\title{
Twitter en los juicios: Una revolución en la información periodística de tribunales
}

\author{
Recibido: 03 de septiembre de 2013 \\ Aceptado: 01 de mayo de 2014 \\ Publicado: 28 de noviembre de 2014
}

\author{
Ana Azurmendi \\ aazur@unav.es
}

Universidad de Navarra (España)

Resumen: El creciente uso de dispositivos electrónicos, como las tablets y smartphones, para la cobertura de juicios ha tenido un fuerte impacto en el trabajo de los periodistas de tribunales. La posibilidad de captar imágenes sin que sea necesario mover una cámara de televisión dentro de la sala de juicio, o aún mejor, ser capaz de transmitir noticias, percepciones y opiniones durante las sesiones gracias a las redes sociales (sobre todo Twitter) ha cambiado no sólo aspectos de la cobertura periodística, sino que también ha supuesto una serie de beneficios y de riesgos para la misma administración de justicia. En ese sentido, a finales de la década del noventa, el juicio a O. J. Simpson (Estados Unidos) fue un antecedente para el debate en torno al acceso de cámaras de televisión en la corte. En la actualidad, el juicio a Oscar Pistorius (Sudáfrica) se configura como un campo de pruebas para contemplar los cambios que Twitter ha introducido en la cobertura periodística judicial.

Palabras clave: Periodismo judicial, redes sociales, Twitter, juicio Oscar Pistorius.

\begin{abstract}
The increasing number of electronic mobile devices like tablets and smartphones, with capacity to tape and report audiovisual contents, has made a considerable impact in reporter's work. The possibility of taping live images without having to move a TV camera in courtroom, o even better, being able to transmit news, perceptions or opinions during judicial proceedings by Social Media (over all Twitter) not only has changed the way in which journalists work, but it has also suppose a series of benefits and risks for the judicial proceeding itself. In the same way that in the late 1990s, the O. J. Simpson trial (USA) was the reference for the debate on the access of TV cameras in Courts, today, the Oscar Pistorius trial (South Africa) is the field of proofs to visualize those changes that Twitter has introduced in the journalistic coverage of Courts.
\end{abstract}

Key words: Judicial Journalism, Social Media, Twitter, Oscar Pistorius Trial. 


\section{Introducción}

El interés de los medios de comunicación por los juicios ha existido siempre. Es lógico que sea así puesto que, en muchos casos, se trata de informar sobre el momento final (el enjuiciamiento) de sucesos que han ocupado espacio en periódicos, radio, televisión y versiones online en Internet. Es evidente que no todos los procesos judiciales interesan del mismo modo. Sólo algunos, entre los miles que tienen lugar cada día en un país, llaman la atención de periodistas y ciudadanos. Conseguir informar de una forma equilibrada sobre los juicios que se han denominado de "alto perfil" tiene innumerables dificultades, pero es imprescindible el ejercicio de un buen hacer informativo ad hoc para evitar uno de los peligros mayores del periodismo de tribunales: los juicios paralelos.

Durante los años noventa del siglo XX, el debate sobre la relación entre medios de comunicación y tribunales tuvo como centro de atención la presencia de las cámaras de televisión en las salas de juicio ${ }^{1}$. De hecho, aún hoy, son relativamente pocos los países que permiten a las televisiones retransmitir sesiones de un proceso judicial ${ }^{2}$. Internet y la tecnología digital han supuesto una revolución también en este campo del periodismo de tribunales, de manera que, en la actualidad, la controversia se ha trasladado a la utilización de tabletas, smartphones y otros dispositivos electrónicos, durante la celebración de los juicios. Con un añadido: el fenómeno de las redes sociales. Porque el cambio de una cobertura de televisión a una cobertura a través de Twitter no consiste sólo en la instantaneidad o la difusión multimedia que con este último medio puede lograrse, sino en las posibilidades de modos de difusión que facilita: amateur y profesional, informativa y de opinión, con interactuación con la audiencia, integrada con la televisión o los medios online, siempre con una capacidad multiplicadora inimaginable con anterioridad.

El desarrollo doctrinal sobre las ventajas e inconvenientes del uso de las redes sociales en el periodismo de tribunales, sobre todo de Twitter (ya sea en su forma aislada o en combinación con otros medios de comunicación digitales) todavía es muy escaso. Por esta razón, en este estudio se tratará de fijar un status quaestionis de las reflexiones publicadas sobre la materia, aunque, como se verá, la mayoría de los trabajos existentes pertenecen al ámbito divulgativo y profesional de los blogs y sites de Internet y son aún muy pocos los publicados en las revistas académicas.

\footnotetext{
1 En Europa, los estudios más representativos han sido, entre otros, los de Pico y Junoy (2001), Sparks (1992), Schlesinger y Tumber (1994), Pradel (1986), Garapon (1996), Hassemer (1990, ápud Brack y otros), Lorz (1996), Zumbansen (2001), Plotino (2001) y Toschi (1988). En Estados Unidos destacan Barber (1989), Gebner (1989, ápud Barber), Borgida, DeBono y Buckman (1990), O’Callaghan \& Dukes (1992), Harris (1993), Hardaway y Tumminello (1996), Greenhouse (1996), Sager y Frederiksen (1996), Arenella (1996 y 1997), New York State Committee to Review Audiovisual Coverage of Court Proceedings (1997), Wermiel (1998), Cohn y Dow (1998), Mehrkens y otros (1999).
}

2 Consultar la regulación vigente en los países europeos y en los Estados Unidos en Azurmendi (2005: 135-178). 
Frente a estas aproximaciones tentativas al fenómeno de las redes sociales en la cobertura periodística de juicios, el caso "Pistorius" 3 ha mostrado la trascendencia de Twitter. No sólo ha confirmado todas y cada una de las consecuencias que los analistas anunciaban de introducirse esta red social en el trabajo del reportero, sino que ha roto las expectativas iniciales ocasionando una auténtica revolución en la forma de reportear desde las salas de juicio.

\section{Preámbulo: un problema añadido, el uso de redes sociales por parte de los participantes en un juicio}

Desde inicios de los años noventa, con el acceso de las cámaras de televisión a las salas de juicio se han planteado, todavía hoy se plantean, situaciones negativas a las que tanto los jueces como los medios de comunicación han tenido que hacer frente, como son el posible efecto intimidatorio en los participantes en el proceso judicial, el tratamiento sensacionalista en la nota televisiva de tribunales o el juicio paralelo en los medios de comunicación. Estas dificultades son graves pero su efecto se limitaría a un tipo de interferencia externa en el proceso judicial. Pero Internet está ocasionando otro tipo de situaciones distorsionadoras de la administración de justicia, de muy distinto cariz, que proceden de la estructura misma del proceso judicial.

\subsection{Las primeras reflexiones}

Fue en 2009 cuando cundió la alarma de que las redes sociales (Twitter, Facebook, etc.) estaban cambiando las normas y los usos en las salas de juicio, y no tanto en los periodistas que cubren tribunales como en los participantes en el proceso judicial. Así lo manifiesta Joseph Rosenbaum, sobre la decisión de numerosos jueces locales y de distrito en Estados Unidos de sustituir la orden a los miembros del jurado de "no se comunique y no lea los periódicos" por la de "no navegue por Internet, ni se comunique por las redes sociales" (ápud Sullivan, 2010).

Según Rosenbaum, la situación actual es transitoria, en el sentido de que aún no se han adoptado medias de carácter general para el uso de Internet y redes sociales dentro de las salas de juicio (ibídem). Pero quizá, lo más significativo de lo que está ocurriendo es que la mayoría de los ciudadanos no comprende por qué deberían limitarse para los juicios el uso de redes sociales, ni por qué los jurados y los jueces sólo pueden contar con las pruebas mostradas en el juicio, cuando Internet permite acumular muchas más evidencias, de signo muy diferente. Es decir: un primer efecto constatado del uso de redes sociales en la cobertura de juicios ha sido el de una percepción distorsionada del modus operandi de los procesos judiciales.

3 Oscar Pistorius, de 27 años, deportista sudafricano convertido en icono en las Olimpiadas de Londres 2012, donde, con sus prótesis biónicas en las dos piernas (amputadas cuando apenas tenía un año), participó en las semifinales de 400 metros. Su imagen se ha utilizado para publicidad de marcas internacionales de primer nivel. El día de San Valentín de 2013 mató a su novia. Desde el 3 de marzo de 2014, se enfrenta al juicio en el que está acusado de homicidio voluntario. 
Esta cuestión afecta no sólo a los miembros de los jurados (ciudadanos en principio sin una preparación jurídica) sino también a los abogados que cada vez más acuden a MySpace, Facebook y Twitter para obtener evidencias sobre los casos y sobre las personas que actúan como testigos, bien para recusar a los miembros del jurado o, incluso, al mismo juez (ibídem). De hecho, el Judicial Ethics Advisory Committee (2009) de Florida, en Estados Unidos, publicó una opinion sobre el tema, señalando que los jueces del Estado de Florida no deben de tener "amigos" en las redes sociales entre los abogados que pueden encontrarse ante ellos en los tribunales.

Abunda la autora, "un testigo de un caso de conducción imprudente por ingesta de alcohol puede perder credibilidad si ese mismo testigo aparece en un video de YouTube conduciendo mientras se toma una cerveza; un juez comprometería su imparcialidad si aparece como 'amigo' en la cuenta de Facebook de un abogado litigante en un caso en el que él esté actuando. Y por otra parte, ¿seguro que los perfiles publicados en MySpace o Facebook de un imputado o un demandado en vía civil no aportan nada a abogados, jueces, jurados?". En este sentido son significativos los casos juzgados en Reino Unido ${ }^{4}$, en los que miembros de jurados fueron condenados por un uso inapropiado de Internet y las Redes Sociales durante los juicios en los que participaron.

\subsection{Los casos Joanne Fraill y Jamie Sewart (2011) y Theodora Dallas (2012) en el Reino Unido}

Reino Unido ha sido el escenario de las primeras sentencias condenatorias por un uso ilícito de las redes sociales e Internet en el transcurso de un juicio. En el primer caso (2011), el juez del proceso en el que Joanne Fraill era miembro del jurado y Jamie Sewart una de las acusadas, pudo probar (probablemente porque él mismo realizó búsquedas en Internet sobre las personas involucradas en el juicio) que Joanne Fraill había estado comunicándose a través de Facebook con la acusada Jamie Sewart, así se expresa en la sentencia que condena a Fraill, en el n. 12:

"Mientras estaban en un receso, el 4 de agosto, al juez Lakin le pareció que un miembro del jurado había estado en contacto en Facebook con Sewart, comentando que le había gustado que Sewart hubiera sido exculpada porque ella 'había estado a su favor durante todo el camino'. Sugirió también que fue una pena que Sewart no hubiera estado en la sala cuando se dieron los veredictos sobre Knox, porque se había perdido 'la expresión de gozo' de la cara de Gary".

\footnotetext{
4 Los casos son HM Attorney General vs. Joanne Fraill y Jamie Sewart (2011) y HM Attorney General vs. Theodora Dallas (England and Wales Court of Appeal (Criminal Division) Decisions, 2011 y 2012), los cuales se analizarán en el siguiente apartado.
} 
Joanne Fraill había utilizado un seudónimo en Facebook. Las investigaciones sobre las conversaciones entre esta miembro del jurado y la acusada muestran su falta de comprensión sobre el papel del jurado y sobre el proceso judicial en su conjunto. En referencia a este punto, la sentencia, en su n. 15 incluye también:

"Se investigó hasta dónde había llegado la comunicación entre Fraill y Sewart. Después de que se exculpara a Sewart de todos los cargos contra ella, la primera comunicación la hizo Fraill. De acuerdo con la prueba aportada por Sewart [...], Fraill envió le envió un mensaje de e-mail a su Facebook diciendo 'tú me debes conocer, he llorado bastante contigo'. Fraill había abierto una cuenta en Facebook con el nombre de Jo Smilie. Sewart aceptó ser su amiga a las 18,30. Sewart respondió, y vio que la cuenta de Jo Smilie tenía como amiga la cuenta de Joanne Fraill en la que aparecía su fotografía. Sewart inmediatamente la reconoció como uno de los miembros del jurado que recientemente le habían exculpado, y se dio cuenta de que el mensaje provenía de un miembro del jurado. Sewart entonces comenzó a chatear con Fraill utilizando el servicio de mensajería instantánea de Facebook. Pero comenzó a preocuparse por el hecho de estar comunicándose con un miembro del jurado. La conversación tuvo lugar entre las 18,31 y las 19,07’.

Los argumentos aportados por la sentencia (n. 29) reflejan de alguna forma las dificultades que Internet puede suponer para la Administración de Justicia:

"Los jueces, como cualquiera, están al tanto para utilizar las nuevas tecnologías en el curso de su trabajo. Internet es un medio moderno de comunicación. La tecnología moderna, y los medios de comunicación, evolucionan a una velocidad cada vez mayor. Sabemos que acudir a Internet es un hábito arraigado en muchas personas, y no cabe duda de que ese hábito crecerá. Pero debemos hablar de forma clara. Insistimos, incluso aunque suponga repetirlo muchas veces, en que si los miembros de un jurado hacen sus investigaciones sobre aspectos de los juicios en los que participan, el sistema del jurado tal y como lo conocemos, una herramienta preciosa para la administración de justicia penal en este país, será seriamente dañado, y lo que es peor, la confianza pública de la que depende será socavada".

La sentencia finalmente concluye (n. 29):

"La revolución de los métodos de comunicación no pueden cambiar estos principios esenciales. El problema, por lo tanto, no es Internet: el problema potencial surge de las actividades de los miembros del jurado que ignoran los principios establecidos desde hace mucho tiempo y que son los que aseguran el derecho de todos los ciudadanos a un juicio justo. Fraill es, tal y como ha 
admitido, culpable de desacato porque como miembros del jurado se comunicó con Sewart vía Internet y mantuvo una discusión online sobre el caso cuando las deliberaciones del jurado todavía no habían terminado y los veredictos no se habían dado. En el curso de la discusión dio a Sewart información sobre la situación de las deliberaciones del jurado. Esta conducta es contraria a lo previsto en la sección 8 de la Ley de 1981 y desobedeció las claras e inequívocas series de directrices dadas por el juez prohibiendo tal conducta. Es también culpable de desacato al tribunal por realizar la investigación en Internet sobre los acusados en el juicio criminal en el que fue jurado, con el propósito de obtener más información de posible relevancia para los asuntos del juicio".

Por estas razones, Frail fue condenada a 8 meses de prisión. En el segundo de los casos, el de Theodora Dallas (2012), en la sesión judicial en la que se le declaró imputada (referencia en HM Attorney General v. Theodora Dallas n. 12 y ss.) se recordaron algunas de las normas sobre el acceso a Internet que se habían dado al jurado en el que Theodora Dallas había tomado parte (n. 14):

"No vaya a Internet. Usted probablemente habrá leído en las últimas semanas sobre un miembro del jurado que acudió a Internet; entró a Facebook y se derivaron problemas muy serios para este miembro del jurado. Estoy seguro de que no quieren que les ocurra nada de eso. Por lo tanto, la norma es -y se dice a todos los jurados- que no sólo no deben discutirlo, sino que no deben ir a Internet; no intente y no realice ninguna investigación por su cuenta; no discuta sobre el tema en Facebook; no tuitee sobre el caso; ni haga ninguna otra cosa parecida. Simplemente, una vez que abandone esta estancia, no hable o trate de esto de ninguna manera con nadie".

A pesar de la advertencia, Theodora Dallas realizó una investigación en Internet sobre el caso que, en aquel momento, todavía estaba juzgándose. Accedió a las noticias publicadas por los periódicos sobre el acusado, y habló con el resto de los miembros del jurado sobre lo que había encontrado. Fue condenada también a 6 meses de prisión.

Son dificultades que no causan sorpresa puesto que Internet forma parte de la vida de muchas personas, para informarse, para entretenerse, para hacer negocios, para comunicarse, para acceder a servicios, contratarlos, etc. Y, seguramente, lo que causa sorpresa para quienes de forma puntual son llamados a formar parte de un jurado, es que sólo las pruebas presentadas en el juicio deben contar para establecer su decisión sobre el caso. Hasta el punto de que autores como Murdoch (2012) han propuesto una reforma del Sistema de Jurado al observar las dificultades que Internet y las redes sociales plantean: 
"El nombramiento de un tribunal independiente que se nombre en cada juicio para estar presente en la deliberaciones del jurado. Un Supervisor del Jurado (Jury Monitor) que no jugaría ningún papel en las deliberaciones y cuya competencia sería la de actuar sólo si se observa o se denuncia alguna conducta errónea".

\section{El uso imparable de las redes sociales por parte de los periodistas para la cobertura informativa de juicios: el caso Pistorius}

Junto a la posible interferencia de las redes sociales en el dinamismo interno de un juicio, está la dimensión externa del mismo: es decir, el cambio que estas formas de comunicar introducen en el relato periodístico de los procesos judiciales. En un informe judicial (Utah State Courts, 2012) se apunta que la tendencia imparable de los periodistas de tribunales es la de tuitear desde las salas de juicios, a pesar de que no en todos los países, desde luego no en todas las salas de juicios, esté permitido.

El último ejemplo notorio de este hecho ha sido el juicio de Oscar Pistorious, sudafricano, atleta olímpico, a pesar de una grave minusvalía que exigió la amputación de sus piernas y su sustitución por unas prótesis biónicas. Acusado de matar a su novia, la modelo Reeva Stemkamp. Un caso que suscita la atención de los medios de comunicación y de los ciudadanos en todo el mundo, debido a la relevancia internacional que tuvo el atleta por su hazaña en las Olimpiadas de 2012. El caso Pistorious es (para la cobertura de tribunales con redes sociales) lo que el caso O. J. Simpson ${ }^{5}$ fue para esa misma cobertura con televisión. La novedad principal ha consistido en que estaba permitido a los periodistas utilizar Twitter dentro de la sala de juicio, a la vez que operaban cámaras fijas de televisión, con una retransmisión televisiva de únicamente 30 segundos de diferido.

La africana $S A B C$ Digital News y los canales internacionales $B B C$ (Reino Unido) y $C N N$ (Estados Unidos) han mantenido una cobertura en directo, en la que han combinado las imágenes y sonido de la sala de juicio, con los tuits de sus corresponsales presentes en el juicio. $B B C$ y $C N N$ tienen, además, una web específica donde puede leerse toda la información al respecto, videos de resumen de las sesiones anteriores, se reproducen los tuits de los periodistas en tiempo real, al mismo tiempo que se ofrecen las imágenes de televisión del momento. Mientras que YouTube alberga todos los vídeos de la retransmisión de la televisión africana, con miles de comentarios de usuarios en cada uno de ellos.

En Sudáfrica se espera que el caso sea el acontecimiento del año 2014 en Twitter, y así se están midiendo las menciones y el número de cuentas que siguen el juicio: 19809 menciones de 9213 cuentas en el primer día (Mawson, 2014). Los datos reflejan un nuevo

5 El juicio televisado del actor y exfutbolista O. J. Simpson por la muerte de su exmujer y su amante, celebrado en 1995, originó tal controversia que se emprendió la reforma de la ley de California sobre el acceso de las cámaras de televisión a las salas de juicios ( $c f$. Azurmendi, pp. 171 y ss.) 
acontecimiento mediático en el que Internet y las redes sociales han obtenido protagonismo absoluto (Granger, 2014). A continuación, se enumeran algunas conclusiones que permiten valorar este alcance:

- $\quad$ Entre el 9 de febrero y el 10 de marzo de 2014 se generaron 893957 posts (figura 1) en las redes sociales durante el juicio.

Figura 1: Posts generados en redes sociales durante el juicio a Pistorius.

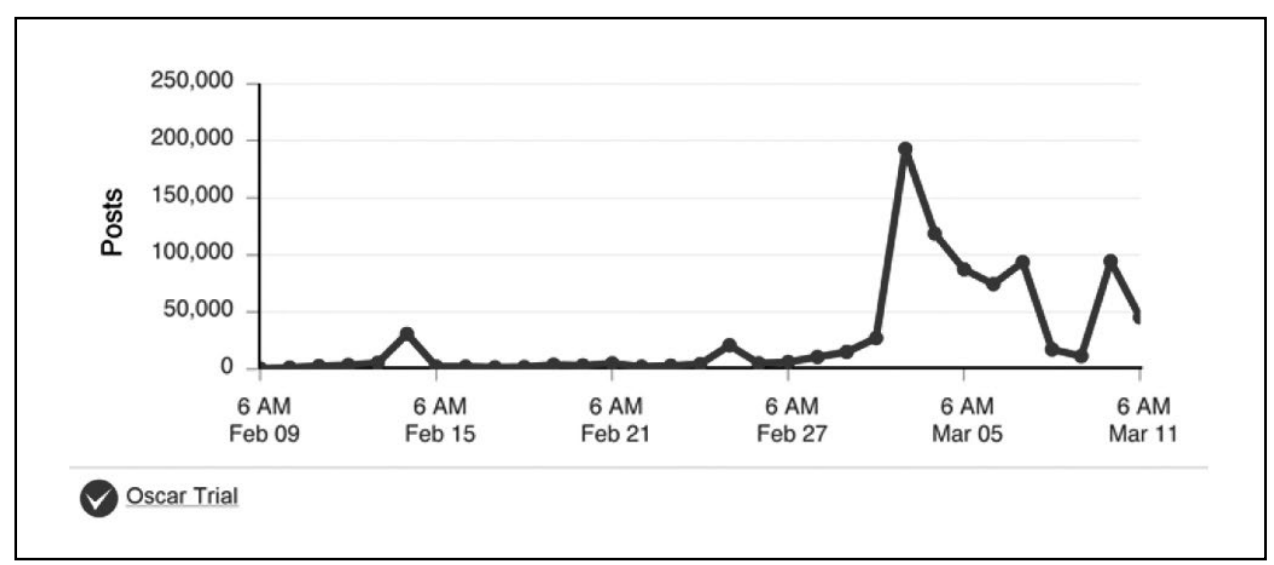

Fuente: Granger (2014).

- Twitter es el canal social dominante, con hastags (\#OscarTrial, \#OscarPistorius) pero también los nombres de los abogados, fiscal, juez y testigos se convirtieron en tendencias en Sudáfrica a medida que el juicio se desarrollaba. Un $87.2 \%$ de todas las conversaciones sobre el proceso han tenido lugar en Twitter, con más de 780000 tuits en un mes. Mientras que Facebook ha acaparado un 3.5\% de todos los post.

- Curiosamente, aunque el juicio está teniendo lugar en Sudáfrica, la mayoría de las conversaciones online se han generado en Estados Unidos, con un 45.7\% de todos los post, con Sudáfrica en segundo lugar con un $21.6 \%$ y Reino Unido con un $7.3 \%$.

- La mayoría de los post compartidos una vez comenzado el juicio (40.4\%) lo han sido entre jóvenes de 20 a 34 años, seguidos por los del grupo inmediatamente mayor de 25-44 años (20.5\%).

- Las cuentas más seguidas de Twitter también se miden: llama la atención que en los primeros puestos están los medios de comunicación (figura 2) antes que las cuentas personales de los corresponsales. 
Figura 2: Cuentas de Twitter más seguidas durante el juicio a Pistorius.

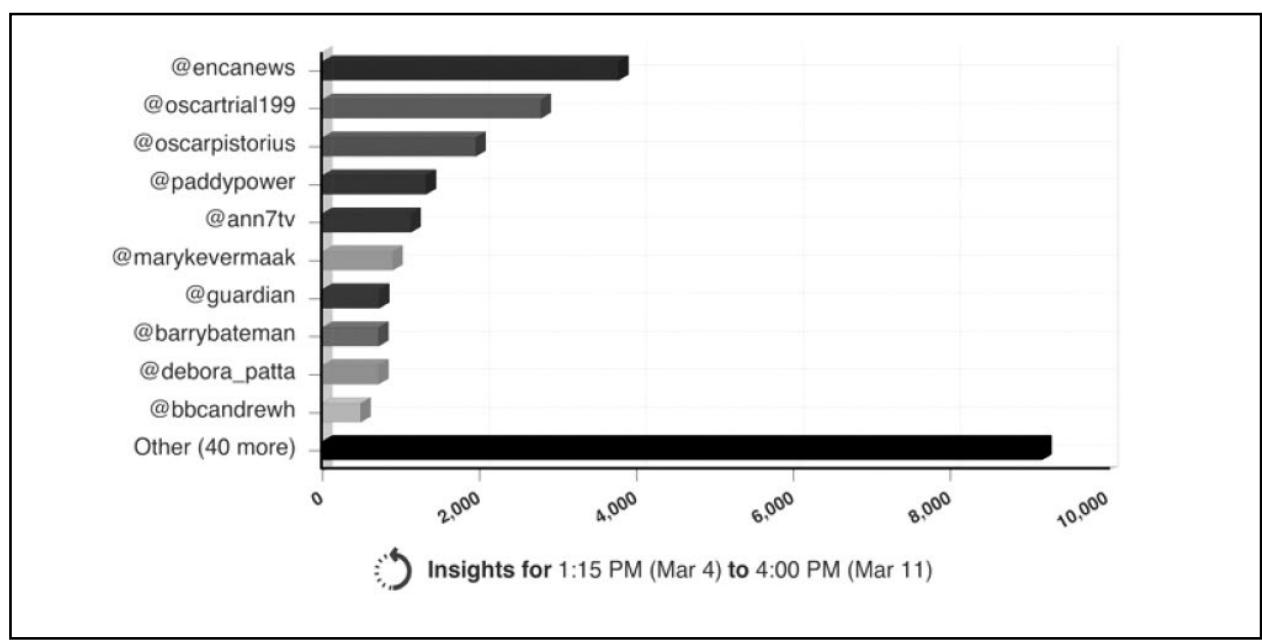

Fuente: Granger (2014).

Spillane (2014) aporta más datos sobre la cobertura del caso Pistorius: mientras que el canal africano de 24 horas de seguimiento del juicio se ha convertido en el primer canal de noticias, con cerca de doscientos mil espectadores en los picos de audiencia, durante la primera semana, en Twitter ha habido una auténtica "explosión de cobertura". Decenas de reporteros en la sala de juicio han subido post sobre cada detalle de lo ocurrido ("desde el color del traje del acusado a la calidad del trabajo de la policía o el humor de la juez").

La intensidad de la nueva cobertura ofrecida por las redes sociales se manifiesta en momentos como el del 24 de marzo de 2014, donde en dos minutos, los corresponsales escribieron 50 tuits con el hashtag \#OscarPistorius, que fueron distribuidos a cerca de 1.2 millones de cuentas con los retuits incluidos.

Pero no se trata sólo de la cantidad de personas que siguen el juicio gracias a las redes sociales, o la distinta franja de edad a la que se llega, sino de un fenómeno con muchas consecuencias, sobre las que apenas ahora se comienza a reflexionar. Anton Harber explica: "lo más chocante es cómo se debate en Twitter sobre cada aspecto del juicio y sus implicaciones casi inmediatamente", en su opinión "está cambiando toda la ecología mediática" (ibídem).

Al visualizar la web sobre el juicio de Pistorius, en el portal electrónico de la $B B C N e w s$ (2014a), en su edición internacional de África, es notorio que Internet y las redes sociales han desplazado a la televisión. El esquema en el que se integran (por este orden) tuits, fotografías y vídeos del juicio es el siguiente en orden, cronológicamente descendente (figura 3): 
Figura 3: Despliegue de contenidos del caso Pistorius en la $B B C$.
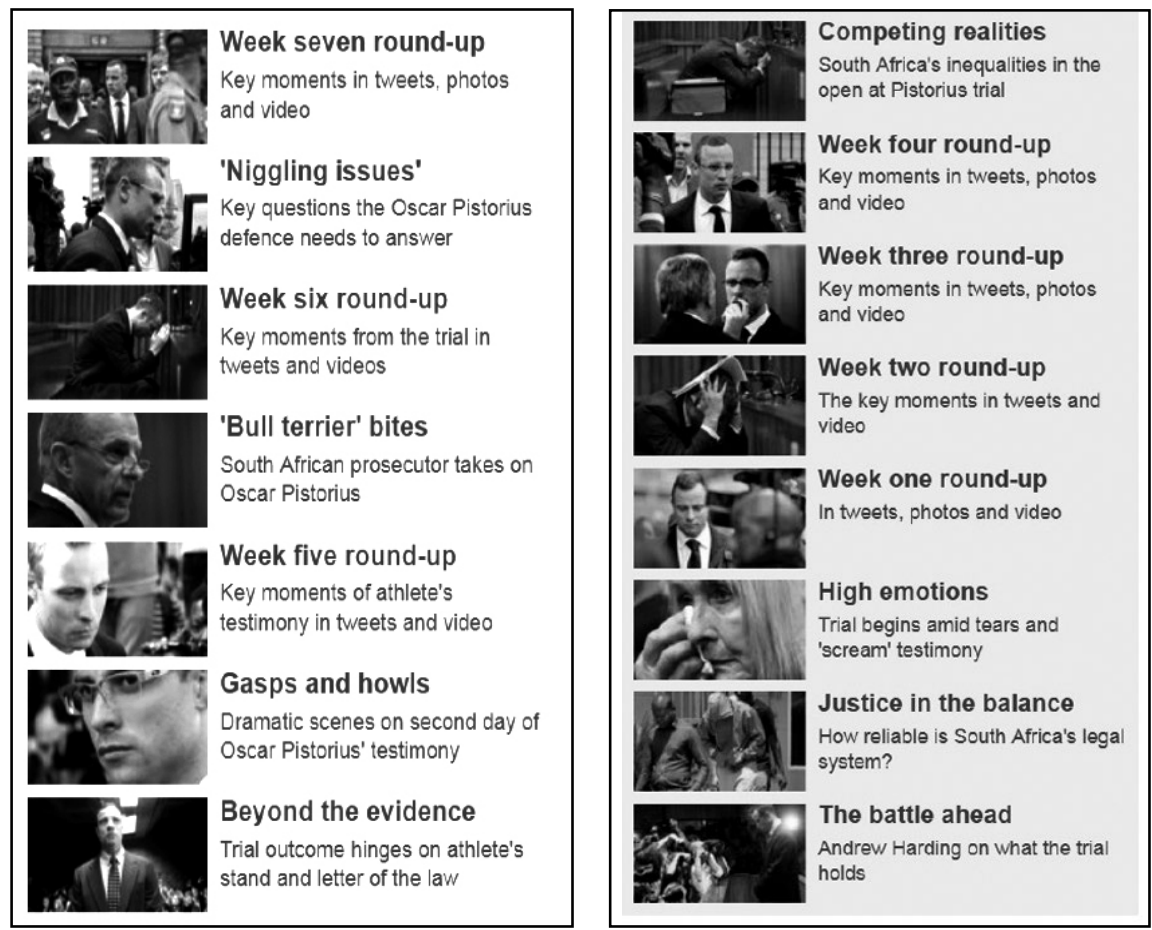

Fuente: $B B C$ News (2014a).

Con una introducción del reportero en el que se apunta la repercusión de la retransmisión del juicio en los medios de comunicación y redes sociales:

"[La Juez, Thokozile Matilda Masipa] estará ante un intenso escrutinio -de los medios de comunicación, del gobierno preocupado por la reputación internacional de Sudáfrica, de los activistas que destacarán los increíbles niveles de violencia de género en este país, de la policía que aplica la justicia a su manera y del público que tiene su propia visión sobre uno de los más icónicos héroes del deporte" (ibídem).

Y donde añade estas inquietantes preguntas sobre la Juez, si lo que desea hacer es una crónica periodística de tribunales:

“Elegirá [la juez] -¿debe hacerlo?-hacer un juicio ejemplarizante del caso Pistorius?

¿Qué señales enviaría al público si Pistorius quedara en libertad? 
Si el atleta es declarado culpable con el cargo menor de homicidio, ¿Pistorius debería ir a la cárcel o una multa junto con una suspensión de sentencia sería lo más apropiado?

¿Cómo encontrar el equilibrio adecuado entre el sentido de la justicia y un procedimiento judicial justo?" (ibídem).

En lo que se refiere a los tuits publicados en la página web de la $B B C$, y que previamente han sido también retransmitidos en directo, en un banner lateral a las imágenes que llegaban de la sala de juicio (figura 4), su contenido y su tono es de un perfil bajo, limitado a lo informativo:

Figura 4: Aparición de tuit en portal de la $B B C$.

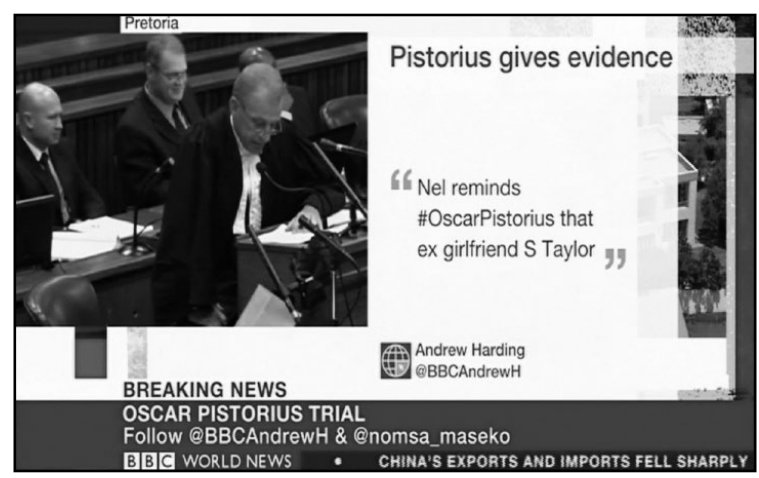

Fuente: $B B C$ News (2014a).

La cobertura de la televisión estadounidense $C N N$ ha sido muy semejante a la de la $B B C$, en esa combinación de retransmisión televisiva que integra los tuits de sus corresponsales en la sala de juicio y que llama, a su vez, a seguir el blog sobre el proceso a Pistorius en Internet:

Figura 5: Aparición de tuit en portal de la $C N N$.

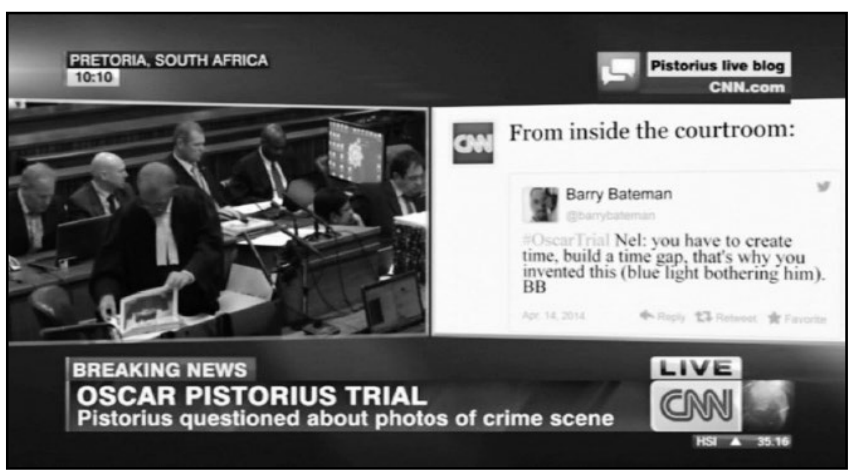

Fuente: $C N N(2014)$. 
Con tuits publicados en los que no sólo se transmite lo que ocurre dentro de la sala de juicio, sino también declaraciones, fuera del palacio de justicia, de familiares, como esta de Arnold Pistorius, tío del acusado:

"Como familia nos sentimos confortados por el transcurso y el detalle de este juicio y el compromiso de la Juez Masipa, utilizando todos los medios posibles para asegurar un juicio justo. Se trata de que haya un juicio justo. Algo que reafirma nuestra confianza en el sistema de justicia de Sudáfrica".

Los tuits, tanto en la $B B C$ como en la $C N N$ han cumplido con la misión de hacer titulares rápidos sobre lo que estaba aconteciendo en la sala, con la excepción de algunos momentos señalados, por ejemplo, al inicio de las sesiones, cuando se describía el atuendo del acusado, sus movimientos o cualquier gesto que pudiera expresar su estado de ánimo. De hecho, donde quizá mejor se refleja la aportación de Twitter a la crónica de tribunales es en los resúmenes semanales de las sesiones del juicio, hecha exclusivamente con una selección de los tuits de los corresponsales combinada con videos y fotografías:

Figura 6: Seguimiento del juicio en el portal de la $B B C$.

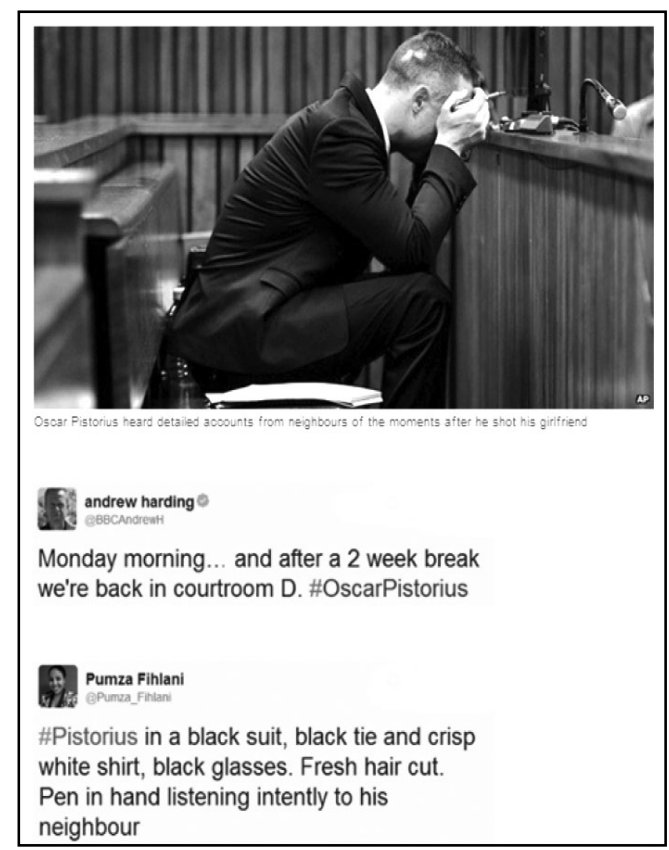

Fuente: $B B C$ News (2014b).

En las dos cadenas internacionales de televisión, la retransmisión televisiva (junto con los tuits) se interrumpía para emitir las noticias (figura 7) que comenzaban precisamente con la información de resumen de la sesión del juicio: 
Figura 7: Cobertura televisiva del juicio en la $B B C$.

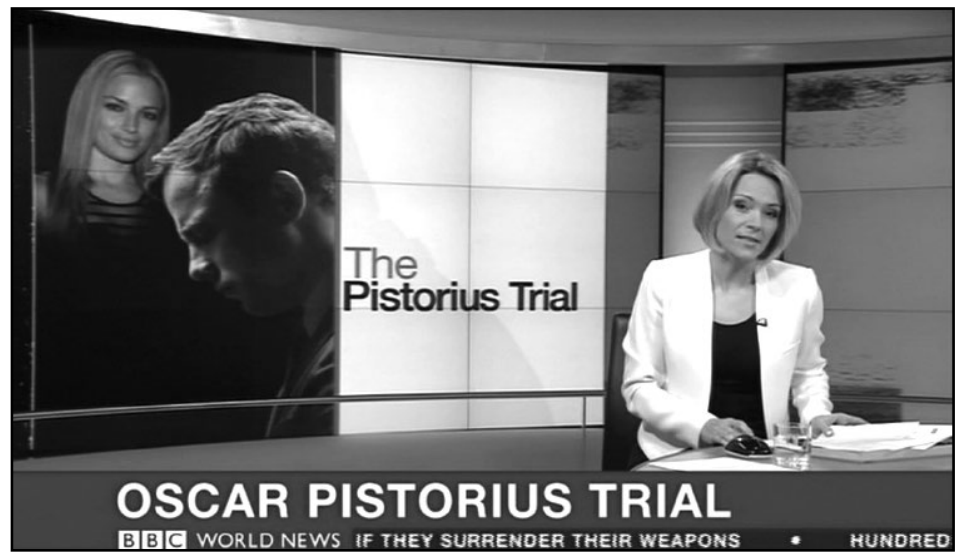

Fuente: $B B C$ News (2014a).

Un procedimiento que la segunda televisión internacional que ha retransmitido el juicio, la CNN (figura 8), también ha seguido:

Figura 8: Cobertura televisiva del juicio en la $C N N$.

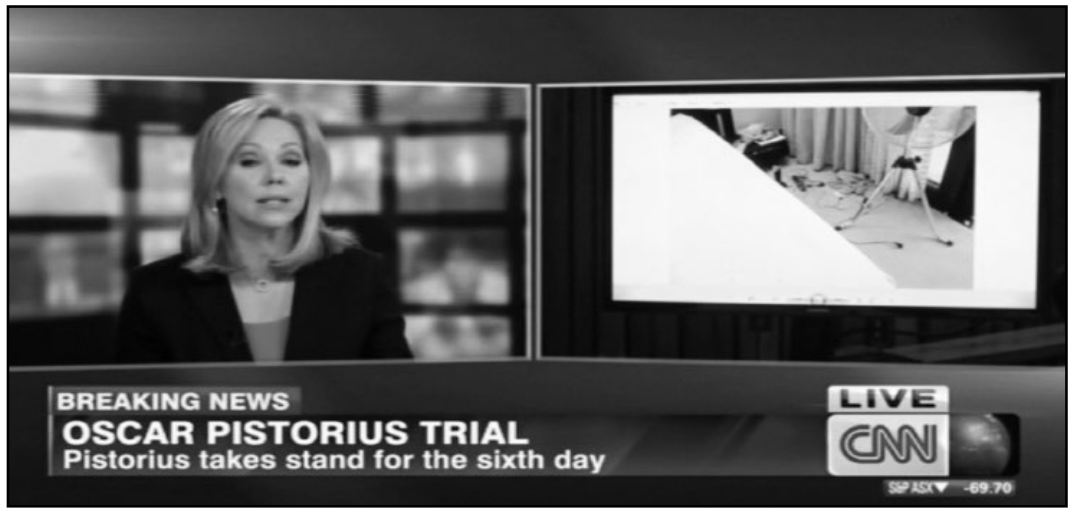

Fuente: $C N N(2014)$.

Pero la importancia de Twitter en este juicio ha ido mucho más allá de una transmisión integrada con y en la televisión. Como destaca Soares (2014), "esta red social se ha convertido en un auténtico 'jurado virtual' del caso", en el que usuarios de todo el mundo han intervenido expresando sus valoraciones y opiniones acerca del juicio, de la actuación de la Juez, del Fiscal, del mismo Pistorius, etc. algo a lo que "no serán inmunes ninguno de ellos". Más aún, en un reportaje de la web de la $C N N$ sobre el juicio, la periodista se ha detenido a comentar algunos de los tuits que han sido tendencia en Twitter (figuras 9 y 10), los cuales (en su opinión) parecen ser los más representativos de los cientos de miles que se han transmitido en la red social: 
Will Black (figura 9):

- "No sé si Oscar \#Pistorius es culpable o no pero que esté llamando a todo el mundo 'my lady' está empezando a ser irritante"'.

Figura 9: Repaso y análisis de tuits en la $C N N$.

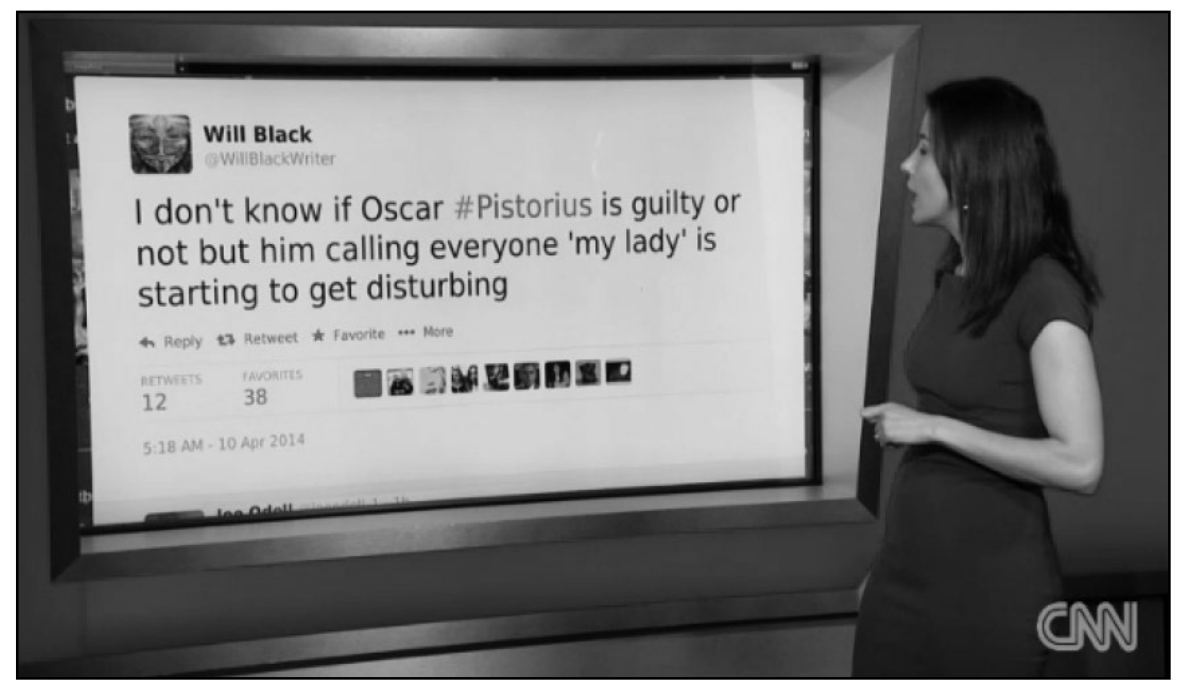

Fuente: Soares (2014).

Mandy Ndubana:

- "Oscar sólo recuerda lo que quiere y olvida lo que quiere olvidar, pero en este caso tu no puedes matar y elegir olvidar \#OscarPistorius" "7.

James Hall:

- "No pinta bien para \#Oscar Pistorius! Interrogatorio brutal"

Mogale Motau (figura 10):

- “Creo que la Juez Masipa admira secretamente o es una fan

6 "I don't know if Oscar \#Pistorius is guilty or not but him calling everyone 'my lady' is starting to get disturbing".

7 “Oscar Just remembers what he wants and forgets what he wants to forget, but in this case you cant't kill and choose to forget \#OscarPistorius".

8 "Outlook is not good for \#OscarPistorius! Brutal cross examination". 
de Oscar Pistorius es muy cuidadosa y atenta con él \#OscarTrial \#Day20"9.

Figura 10: Repaso y análisis de tuits en la $C N N$.

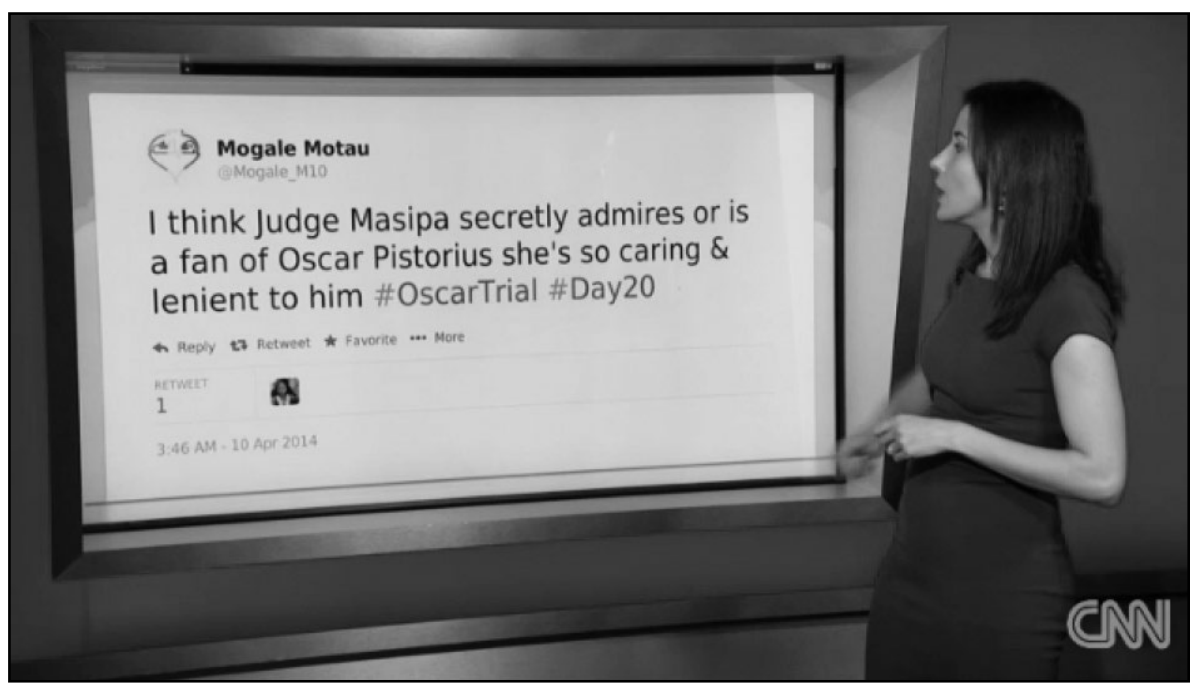

Fuente: Soares (2014).

Mmkobedi Choabi:

- “@barrybateman la juez es muy atenta con Oscar... Hay demasiados 'no sé' me transmite la idea de que tiene ya el veredicto" $"$.

Soares remarca al final que "no hay una única juez del caso Pistorius en una sala de juicio de Pretoria, sino cerca de un millón de jueces del caso en todo el mundo" (ibídem).

Es una nueva forma de cubrir tribunales en televisión, donde el medio de comunicación convencional realza e intensifica el poder multiplicador de la red social, en este caso Twitter, al mismo tiempo que la voz de los ciudadanos alcanza una relevancia inédita. Por otro lado, Internet hace posible volver una y otra vez sobre las noticias del juicio, la retransmisión televisiva, los tuits, los comentarios de los más diversos tipos de usuarios, los videos sobre el juicio que se han subido a YouTube, en una secuencia interminable de información y valoración sobre el proceso judicial.

9 "I think Judge Masipa secretly admires or is a fan of Oscar Pistorius she's so caring \& lenient to him \#OscarTrial \#Day20".

10 “@barrybateman judge is too lenient on Oscar... there are too many 'I don't knows' she is giving me the idea that she has the verdict already". 


\section{Lo específico de Twitter en comparación con la retransmisión televisiva de procesos judiciales: pros y contras}

No sólo en el caso Pistorius, también en otros casos de juicios alto perfil como han sido en Estados Unidos el juicio, en 2012, a William Balfour (acusado y luego condenado por el asesinato de la madre, el hermano y el sobrino de 7 años de la actriz y cantante Jennifer Hudson), o el juicio, en 2013, al Dr. Conrad Murray sobre la muerte del cantante Michael Jackson se ha observado este nuevo fenómeno en la cobertura informativa de tribunales. Pero, junto con la amplitud de la difusión que los casos llegan a adquirir y la participación de público joven en las conversaciones sobre los juicios en las redes sociales, dos notas muy relevantes, se han observado con algo más de detalle los efectos positivos y negativos que, por ahora, parecen derivarse.

Así, en las websites especializadas (de colegios de abogados, de blogs de Derecho de la Comunicación, de Facultades de Periodismo) la discusión se centra sobre permitir o no los dispositivos móviles, particularmente los smartphones y laptops, con los que poder informar desde las salas de juicio. Los argumentos a favor serían muy semejantes a los empleados en los años noventa para apoyar el acceso de las cámaras de televisión a las salas de juicio. Entre ellos, los más repetidos:

- “La naturaleza pública del juicio y el derecho de acceso del público es la principal razón para la cobertura audiovisual y con redes sociales de los procesos judiciales.

- La retransmisión de los juicios tiene valor instructivo para los ciudadanos; en primer lugar, por la cercanía con que se presenta la actividad que tiene lugar en la sala, de forma que se produce una familiaridad con el sistema judicial; en segundo lugar, porque se informa a la ciudadanía de qué conductas son antisociales y el castigo que merecen.

- Elescrutinio público del sistemajudicial que resulta de la retransmisión de los procesos hace que todos los participantes del proceso -juez, abogado defensor, fiscal, acusación, testigos, etc- cumplan mejor su papel" (New York State Committee..., 1997: 38 y 39).

Aunque, además, se añadirían otras ventajas que las nuevas tecnologías (laptops y smartphones) y redes sociales ofrecen:

- Facilitan la movilidad e instantaneidad para captura y diseminar imágenes, sonidos y textos.

- Hacen posible la accesibilidad a todos los usuarios presentes en la sala de juicio, no sólo a los profesionales de la información. 
- Proporcionan una comunicación interactiva constante que promueve la participación ciudadana en asuntos de interés público.

- Los contenidos difundidos por redes sociales pueden alcanzar, por su efecto multiplicador, a un número mayor y también más diverso de ciudadanos ( $c f$. Davey y otros, 2010; Elefant y Black, 2010; Eve y Zurckerman, 2012).

En cuanto a las objeciones con carácter común a la cobertura televisiva y de las redes sociales $^{11}$ :

- "Una sala entregada a los espectadores y a los observadores es una sala que presta menos atención a la justicia" tal y como concluyó el New York State Committee to Review AudioVisual Coverage of Court Proceedings (1997: 38 y 39).

- Necesariamente se produce una distorsión sobre el sistema judicial y los juicios porque el criterio con el que se solicita el acceso de las cámaras y la retransmisión de juicios es meramente sensacionalista (ibídem: 32 ).

- La retransmisión televisiva, y mucho más la información por redes sociales, convierten los juicios en pequeños fragmentos para noticias de la televisión o posts en Internet, algo que crea confusión sobre la realidad de un proceso judicial e incluso sobre el sistema judicial en su totalidad (ibídem: 38 y 39).

Con respecto a los efectos negativos específicos de la cobertura de tribunales vía redes sociales:

- Existe una posibilidad Mayor de difundir errores precisamente por la instantaneidad y por el hecho de que cualquiera $-\mathrm{y}$ no sólo los profesionales de la información- pueden diseminar datos y comentarios sobre el transcurso de un juicio.

- Las redes sociales favorecen una información fragmentada de un proceso judicial, cuando, en realidad, es imprescindible esperar a que el juicio llegue a su fin para que todo cobre pleno sentido.

- En particular Twitter, con el límite de 140 caracteres máximo de un tuit, sólo permite difundir una información muy simplificada.

11 Consultar aportes de Tarm (2012), Beaujon (2012) y Carton (2012). Sobre el acceso de cámaras: Gebner (1989: 15) y Zumbansen (2001). 
- Las redes sociales facilitan que el centro de atención sean aspectos accidentales del proceso -atuendo del encausado, sus reacciones, sus gestos, su forma de hablar, etc y también los de sus familiares presentes en la sala o de los familiares de la víctima, por ejemplo.

- Existe el riesgo de que los juicios se conviertan en un "drama deliberativo", precisamente porque son objeto de una difusión continuada, en el que queda para el final la solución del caso.

- Si un juicio se convierte en trend topic en las redes sociales, sobre el que cualquiera puede verter sus comentarios, se creará un estado de opinión radicalizado pero poco fundado en la verdad procesal, o con el beneficio que debe significar la publicidad para la persona encausada ( $c f$. Tarm, 2012 y Carton, 2012).

Krawitz (2013) destaca la circunstancia de que, gracias a los dispositivos electrónicos móviles, los periodistas pueden escribir sus crónicas sin necesidad de abandonar la sala de juicio; en consecuencia, es más probable que sus relatos periodísticos sean más completos y ajustados a la realidad. Otra de las ventajas señaladas por la autora es el hecho de que esos dispositivos permiten "ver y oír la atmósfera de la sala y educar e informar mejor sobre el trabajo de los tribunales". Argumentos que fundamenta en algunas de las guías que han publicado tribunales como los de los Estados americanos de Utah (Reporters Committee..., 2014) y de Arizona (Newsgathering Committee..., 2010). Desde esta perspectiva, concluye que "de la misma forma que los medios de comunicación se inventan y reinventan, también el sistema judicial debería evolucionar en su relación con los medios de comunicación” (Krawitz, 2013).

En definitiva, Twitter añade beneficios y riesgos para la información periodística sobre el sistema judicial y los juicios. Entraña una mayor cercanía de los ciudadanos hacia los procesos judiciales pero también el peligro de una comprensión distorsionada, incluso banalizada, de la realidad de tribunales. La experiencia del juicio a Oscar Pistorius, aún inacabada (el proceso judicial está todavía abierto en mayo de 2014), muestra que cada uno de los beneficios y de las objeciones señaladas alcanzan una proyección mucho mayor de lo pensado.

\section{La opinión de los periodistas sobre el uso de Twitter en las salas de juicio}

La mayoría de argumentos en el debate del uso de redes sociales en las salas de juicio están relacionados con la posible interferencia en el proceso judicial. En todo caso, la visión de los medios de comunicación se ha centrado hasta ahora en defenderse de las limitaciones excesivas, en su opinión, que les han impuesto muchos jueces para el uso de redes sociales en las salas de juicio.

Existe, sin embargo, otro debate importante sobre qué es exactamente lo que aporta el uso de estos nuevos medios de comunicación al periodismo de tribunales y sobre los cambios 
que introduce en el relato judicial. No se han encontrado informes, ni artículos académicos sobre este tema. Sí algunos blogs y encuestas realizadas en Facultades de Comunicación. Tienen un valor limitado como estudio sobre el tema, pero representan el estadio actual de la reflexión sobre el uso de las redes sociales para el periodismo de tribunales.

Carton (2012), a propósito de la solicitud de un condenado de un nuevo juicio de su causa (2010, caso Allen Stanford, financiero acusado de fraude millonario) con motivo del uso indiscriminado de Twitter en la sala y de la falta de advertencia por parte del juez al jurado de que se mantuvieran al margen de la red social, analiza cómo algunos periodistas consideran que tuitear en estas situaciones entra de lleno en la libertad de expresión reconocida en la First Amendment.

Para Cleirmont (2013) poder tuitear desde la sala de juicio significa que "el proceso judicial será más abierto, transparente y responsable". Y prosigue: "es un reto intentar tomar notas, tuitear, escuchar y observar. He entrado en un ritmo de utilizar Twitter como si fuera un notebook, más que duplicar la información en mis notas escritas”.

Vettese (2012) planteó la siguiente pregunta en Internet: ¿Tuitear desde la sala de juicio puede interferir en el proceso judicial? Las respuestas de 36 estudiantes de periodismo son variadas, pero inciden en su mayoría en tratar el tema desde el punto de vista del reportero y su trabajo, mucho más que en si se puede distorsionar o no la decisión de los miembros del jurado a causa del uso de redes sociales. Se seleccionan algunos de los argumentos publicados:

Jordan:

- "Cuando la gente oye noticias chocantes desea darlas a conocer a todo el mundo Lo que ocurre en las salas de juicio es público y desde ese momento la gente tiene el derecho a compartir la información como lo desee".

Lauren:

- "No sé hasta qué punto es importante mantener al público informado en 'tiempo real' (¿no puede esperar la gente a tener la información completa sobre la historia al final?), pero, en cualquier caso, es una tendencia actual en los reportajes y no pienso que el argumento de la 'historia incompleta' aguante, considerando que siempre ha habido historias incompletas y noticias parciales en la comunicación oral entre personas".

Yasmin:

- "No me parece que el uso de Twitter desde las salas de juicio deba prohibirse. Claro que puede distraer, pero posiblemente menos que un hombre corriendo en la sala. Tuitear es una nueva 
forma de medio, de red social que todavía es muy reciente y todo el mundo está sobrerreaccionando diciendo que puede distraer e interferir mucho. Twitter no es la fuente más confiable de información puesto que en un máximo de 140 caracteres no dan para más, pero las fuentes menos dignas de crédito existen desde hace mucho tiempo".

Lucy:

- "Dependiendo de sobre quién y de qué tipo de juicio hablemos se determinaría si el reportero debe o no informar al público sobre qué es lo que está ocurriendo durante el juicio. Tuitear durante el proceso judicial podría afectar a una percepción injusta por parte del público en un caso porque, con sólo 140 caracteres, los hechos pueden ser fácilmente simplificados al resumirse y cambiarse con el fin de ajustarse al límite. Puede ocurrir que luego la información no tenga que ver con lo que verdaderamente la fuente ha dicho en el caso".

Jocelyn:

- "En un juicio se supone que la persona acusada es inocente hasta que se prueba que es culpable [...] ¿No debería el reportero esperar a poder publicar un reportaje completo de lo que ha ocurrido durante el juicio más que intentar encajarlo todo en 140 caracteres? [...] E incluso pienso que el trabajo de los reporteros es informar al público, yo como audiencia prefiero esperar unas cuantas horas o incluso un día o dos para tener la historia completa que tener distorsionada mi percepción del caso".

Randie:

- "Me parece que es mantener a la gente informada sobre lo que está ocurriendo en la sala. De todos modos, estoy de acuerdo de que algunas cosas se darán fuera de contexto cuando se tuiteen, porque sólo puede hacerse en 140 caracteres. Entonces sí, tuitear podría probablemente dar como resultado una información sesgada".

Carly:

- "Es extremadamente triste vivir en un país tan obsesionado por la gratificación instantánea hasta el punto de que tuitear desde un juicio esté siendo objeto de debate [...] Un malentendido de los hechos por parte del público puede ser desastroso cuando el juez sentencia en un sentido diferente a lo que la gente piensa que 
es la mejor solución. Una difusión continuada por Internet, con constantes actualizaciones desde la sala de juicio puede dañar la autoridad judicial y la verdad del caso. Además, los tuits son para celebridades, adolescentes y resultados deportivos. Si [...] pretendemos que nuestros palacios de justicia sean respetados como lugares de justicia y verdad, debemos evitar que se transformen en un drama elaborado para Twitter.

Ashley:

- "Es cierto que Twitter se ha convertido en una fuente de información principal [...] Los tiempos han cambiado, la tecnología ha avanzado y es bueno intentar mantenerse en constante mejora. Esta mejora hace más fácil que la gente se mantenga informada, pero creo que debe existir un límite para lo que es 'tuiteable'. No todo lo que ocurre en una sala de juicio es adecuado para tus 'seguidores' de Twitter. En nuestros días los cotilleos se extienden como las llamas gracias a Twitter o a Facebook, y honradamente no siento que necesite estar informado sobre quien está flirteando o quién está hablando a mi espalda. Hay información que debe mantenerse privada hasta que está preparada para hacerse pública. Como el Tribunal Supremo ha dicho en el pasado 'lo que transpira en la sala de juicios es propiedad pública'. Los periódicos no deben publicar sus artículos hasta que han sido revisados y editados múltiples veces, entonces ¿por qué querrían noticias que ni se han revisado y que pueden posiblemente desinformar al público? $\mathrm{Si}$, las noticias pueden obtenerse rápidamente con estos nuevos avances, pero puede ser hiriente para cualquiera que esté envuelto en el caso y puede también ocasionar que salga una información falsa. No creo que se deba prohibir tuitear desde la sala de juicio, pero sí que los reporteros deben tener un cuidado extra cuando tuitear información sobre un caso".

Itzel:

- "En realidad, el hecho de que exista un debate sobre si la gente debe tuitear durante un juicio ¡es ridículo! Twitter no debe jugar ese drástico papel en nuestra sociedad -aunque lo esté jugando. No me interesa Twitter, pero existen ciertas limitaciones que no deben ser traspasadas. Lo que ocurra durante el juicio debe permanecer en el juicio. No hay necesidad para que todo el mundo descubra pequeñas historias al instante. Pienso que todavía lo estropearía más. Provoca rumores y altera situaciones que no deberían ser importantes. $\mathrm{Y}$ Twitter hace de ellas un gran tema. No se debería permitir tuitear desde los juicios. Es una forma de respeto y de privacidad". 
Ryan:

- "Tuitear es una manera rápida de obtener información. Hoy todo el mundo tiene Twitter, por lo tanto creo que es la manera más rápida de tener a la gente informada sobre la última novedad informativa".

Ventajas y desventajas percibidas por reporteros jóvenes que manejan las redes sociales como su vehículo de comunicación habitual.

\section{Conclusiones}

El uso de Internet y de las redes sociales en la crónica de tribunales es un fenómeno imparable que ha revolucionado la manera de informar tanto en televisión como en los medios de comunicación online. En cualquier caso, los efectos de Twitter, Facebook y de Internet en general sobre el dinamismo de los juicios, no se derivan sólo de la actividad periodística sino de su influencia decisiva en aspectos internos del proceso judicial, tales como la posibilidad de interactuación instantánea entre sus protagonistas, o de acceso en Internet a una información decisiva que no puede operar en el juicio, tal y como lo muestran los casos Joanne Fraill (2011) y Theodora Dallas (2012) en el Reino Unido.

Ha sido el juicio a Oscar Pistorius, un juicio de alto perfil, el campo de pruebas para visualizar los cambios que las redes sociales, sobre todo Twitter, han introducido en la información sobre procesos judiciales. La retransmisión televisiva (en directo) del juicio ha integrado la crónica de Twitter de periodistas situados en la sala de juicio, pero no sólo eso, en muchas ocasiones han cobrado relevancia tuits de ciudadanos que han comentado las noticias. La televisión ha intensificado el efecto de Twitter, también el efecto de abrir la crónica de tribunales a la participación ciudadana, al dedicar espacio tanto en la televisión como en las websites de las televisiones para presentar, comentar y señalar las tendencias del día de Twitter sobre el juicio. Por otro lado, estas mismas webs dedicadas al proceso judicial (dentro de las webs de las cadenas televisivas que lo han retransmitido) constituyen un fondo documental de vídeos, fotografías, artículos de información y de opinión, y tuits cuya accesibilidad se ha hecho aún mayor gracias a Internet. Resulta difícil prever qué consecuencias tendrán en el desarrollo de los juicios esta sobreactuación informativa, pero lo que es cierto es que se inaugurado una forma distinta de hacer periodismo de tribunales.

\section{Fuentes consultadas}

Arenella, P.

(1997). "Televising High Profile Trials: Are We Better Off Pulling the Plug?" Santa Clara Law Review. Vol. 37, núm. 4, pp. 879-912. Extraída el 23/VI/2014 desde http:// digitalcommons.law.scu.edu/cgi/viewcontent.cgi?article=1463\&context=lawreview 
(1996). "Foreword: O. J. Simpson Lessons". Southern California Law Review, vol. 69, núm. 7.

Azurmendi, A. (2005). "Derecho a la información y Administración de Justicia”. Revista Española de Derecho Constitucional, núm. 75, pp. 135-178. Extraída el 23/VI/2014 desde http://dspace.si.unav.es/dspace/bitstream/10171/15385/1/Ana\%20Azurmendi\%20 Derecho\%20a\%201a\%20Informaci\%C3\%B3n\%20y\%20Administraci\%C3\%B3n\%20 de\%20Justicia.pdf

Barber, S. (1989). News Cameras in the Courtroom: A free Press-Fair Trial Debate. Nueva Jersey: Ablex Publishing Co.

$B B C$ News, sitio web.

_(2014a). "Oscar Pistorius Trial". Extraída el 24/VI/2014 desde http://www.bbc.com/ news/world-africa-26354615

_(2014b, mayo 9). “Oscar Pistorius trial: Week seven round-up”. Extraída el 24/VI/2014 desde http://www.bbc.com/news/world-africa-27346441

Beaujon,A. (2012). "Jerry Sandusky trial starts today, with no tuiting or courtroom blogging”. Extraída el 24/VI/2014 desde en http://www.poynter.org/latest-news/mediawire/176129/ jerry-sandusky-trial-starts-today-with-no-tuiting-or-blogging-from-courtroom/

Borgida, E.; DeBono, K. y Buckman, L. (1990). "Cameras in the courtroom: The effects of media coverage on witness testimony and juror perceptions". Law \& Human Behavior, núm. 14, pp. 489-509.

Carton, B. (2012). "Is Tuiting From the Courtroom by Reporters Too Distracting for Jurors?" Extraída el 24/VI/2014 desde http://legalblogwatch.typepad.com/legal_blog watch/2012/04/is-tuiting-from-the-courtroom-by-reporters-too-distracting-for-jurors.html

Cleirmont, S. (2013, abril 16). "Tuiting from the courtroom has changed the game for reporters". Extraída el 24/VI/2014 desde http://www.thespec.com/news/local/ article/918946--clairmont-tuiting-from-the-courtroom-has-changed-the-game-forreporters

CNN (2014). “Oscar Pistorius trial”. Extraída el 24/VI/2014 desde http://cnnworldlive.cnn. com/Event/Oscar_Pistorius_trial_4?Page $=137$

Cohn, M. y Dow, D. (1998). Cameras in the Courtroom: Television and the Pursuit of Justice. Carolina del Norte: McFarland.

Davey, C. y otros (2010). "New Media and the Courts: The Current Status and a Look at the Future". Conference of Court Public Information Officers, Atlanta.

Elefant, C. y Black, N. (2010). Social Media for Lawyers: the Next Frontier. Chicago: American Bar Association. 
England and Wales Court of Appeal (Criminal Division) Decisions _(2012, enero 23). "HM Attorney General v. Theodora Dallas". Extraída el 23/VI/2014 desde http:/www.bailii.org/ew/cases/EWHC/Admin/2012/156.html _(2011, junio 14). "HM Attorney General v. Joanne Fraill and Jamie Sewart". Extraída el 23/VI/2014 desde http://www.bailii.org/ew/cases/EWCA/Crim/2011/1570.html

Eve, A. y Zurckerman, M. (2012). "Ensuring an impartial Jury in the age of Social Media". Duke Law \& Technology Review, vol. 29. Extraída el 24/VI/2014 desde http://scholarship. law.duke.edu/cgi/viewcontent.cgi?article $=1228 \&$ context $=\mathrm{dltr}$

Garapon, A. (1996). "Eschyle, Kafka, O.J.Simpson: genèse et corruption du rituel judiciaire”. Les Cahiers de Médiologie, núm. 1, pp. 209-219.

Gebner, G. (1989). “Televised Trials-Historic Juncture for our Courts?” En Barber, S. News Cameras in the Courtroom: A free Press-Fair Trial Debate. Nueva Jersey: Ablex Publishing Co.

Granger, L. (2014). "Twitter and beyond: the \#Oscartrial by the social media numbers en Memeburns". Extraída el 24/VI/2014 desde http://memeburn.com/2014/03/twitter-andbeyond-the-oscartrial-by-the-social-media-numbers/

Greenhouse, L. (1996). “Telling The Court's Story: Justice and Journalism at the Supreme Court”. Yale Law Journal. Vol. 105, núm. 6, pp. 1537-1561.

Hardaway, R. y Tumminello, D. (1996). "Pretrial Publicity in Criminal Cases of National Notoriety: Constructing a Remedy for the Remediless Wrong". American University Law Review, vol. 46. Extraída el 23/VI/2014 desde http://www.wcl.american.edu/journal / lawrev/46/hardaway.pdf

Harris, D. (1993). "The Appearance of Justice: Court TV Conventional Television, and Public Understanding of the Criminal Justice System”. Arizona Law Review. Vol. 35, núm. 4, pp. 785-828.

Hassemer, W. (1990). "Der Einfluss der Medien auf das Strafahren aus strafechlichter Sicht”. En Brack, H. y otros (eds.). Der Einfluss der Medien aud das Strafverfahren. Múnich: Instituts für Rundfunkrecht and University of Köln.

Judicial Ethics Advisory Committee (2009, noviembre 17). "JEAC Opinion 2009-20 FLORIDA SUPREME COURT”. Extraída el 23/VI/2014 desde http://www.legalbytes. com/ uploads/file/2009_11_17\%20Florida\%20JEAC\%20Opinion\%202009-20.pdf

Krawitz, M. (2013). "Stop the Presses, but not the Tweets: Why Australian Judicial Officials Should Permit Journalists to Use Social Media in the Courtroom". Flinders Law Journal. Vol. 15, núm. 1.

Lorz, R. (1996). Gerchtsberichtserstattung und Informationsanspruch der öffentlichkeit aus der Sicht deutscher und amerikanischer Verfassungsrechtsprechung. Stuttgart: Boorberg Verlag. 
Mawson, N. (2014). “\#Oscarpistorius hype cause Twitter fatigue en ITWeb". Extraída el 24/VI/2014 desde http://www.itweb.co.za/index.php?option=com content\&view=article\&id=7 1376

Mehrkens, N. y otros (1999). "The Effects of Pretrial Publicity on juror Verdicts: A MetaAnalytic Review”. Law \& Human Behavior. Vol. 23, núm. 2, pp. 219-235.

Murdoch, C. (2012). "The Oath and the Internet". Criminal Law \& Justice Weekly. Vol. 176, núm. 10, pp. 149 y 150. Extraída el 23/VI/2014 desde http://www.1 cor.com/1140/ records/259/CLJW_176_11_Murdoch.pdf con acceso Mayo 2014.

New York State Committee to Review Audiovisual Coverage of Court Proceedings (1997). “An Open Courtroom. Cameras in New York courts”. Nueva York: Fordham University Press.

Newsgathering Committee, Defense Counsel Section, Media Law Resource Center (2010). "Model Policy on Access and Use of Electronic Portable Devices in Courthouses and Courtrooms \& Memorandum in Support for MLRC's Model Policy on Electronic Devices”. Extraída el 24/ VI/2014 desde http://www.azcourts.gov/Portals/74/WIRE/06072012/5 Policies.pdf

O’Callaghan J. \& Dukes, J. (1992). "Media Coverage of the Supreme Court's Caseload". Journalism Quarterly, vol. 69, pp. 195-203.

Pico y Junoy, J. (2001). Problemas actuales de la Justicia penal: los juicios paralelos, la protección de los testigos. Barcelona: Bosch.

Plotino, F. (2001). Il dibattimento nel nuevo Codice di Procedura Penale. Milán: Giufre.

Pradel, J. (1986). Les techniques audiovisuelles, la justice et l'histoire. París: Dalloz-Sirey.

Reporters Committee for Freedom of the Press (2014). Extraída el 23/VI/2014 desde http:// www.rcfp.org/sites/default/files/docs/20121120_133113_cameras_in_court.pdf

Sager, K. y Frederiksen, K. (1996). "Televising the Judicial Branch: in Furtherance of the Public's First Amendment Rights”. Southern California Law Review, vol. 69, n. 7.

Schlesinger, P. y Tumber, H. (1994). Reporting Crime: The Media Politics of Criminal Justice. Oxford: Clarendon Press.

Soares, I. (2014). "Social media reacts to Pistorius trial”. Extraída el 24/VU/2014 desde http:/edition.cnn.com/video/?/video/crime/2014/04/11/lkl-pistorius-virtual-jury-isasoares.cnn\&iid=article_sidebar\&video_referrer $=\mathrm{http} \% 3 \mathrm{~A} \% 2 \mathrm{~F} \% 2 \mathrm{Fedition} . \mathrm{cnn} . \mathrm{com} \% 2 \mathrm{~F} 2$ 014\%2F04\%2F11\%2Fworld\%2Fafrica\%2Foscar-pistorius-trial\%2Findex.html

Sparks, R. (1992). Television and the Drama of Crime: Moral Tales and the Place of Crime in Public Life. Buckingham: Open University Press. 
Spillane, C. (2014). "Pistorius Trial Twitter Coverage Challenging TV”. Extraída el 24/ $\mathrm{VI} / 2014$ desde http://www.bloomberg.com/news/2014-03-25/pistorius-trial-twittercoverage-challenging -tv.html

Sullivan, L. (2010, marzo 10). "Twitter and Facebook Creating Challenges for U.S. Courts". Extraída el 23/VI/2014 desde http://www.mediapost.com/publications/article/124055/\# axzz2RIaIzcoa

Tarm, M. (2012, abril 17). "Courtroom Tuit Ban? Judges, Journalists Clash Over Social Media In High-Profile Upcoming Trial”. Extraída el 26/VI/2014 desde http:/www. huffingtonpost.com/2012/04/17/courtroom-tuit-ban-judge_n_1432686.html con acceso Mayo 2014.

Toschi, A. (1988). Il segretto nell istruzione penale. Milán: Giuffre.

Utah State Courts (2012, abril 10). "Judicial Council Study Committee on Technology Brought into the Courtroom”. Extraída el 23/VI/2014 desde http://www.rcfp.org/sites/ default/files/docs/20121120_133113_cameras_in_court.pdf

Vettese, J. (2012, abril 30). "Social Media in the Courtroom: Can tuiting disrupt a trial?" Extraída el 24/VI/2014 desde http://www.annenbergclassroom.org/speakout/social-mediain-the-courtroom-can-tweeting-disrupt-a-trial

Wermiel, S. (1998). "News media Coverage of the United States Supreme Court". St. Louis University Law Journal, vol. 42.

Zumbansen, P. (2001). "Federal Constitutional Court Affirms Ban of TV-Coverage of Court Proceedings". German Law Journal, vol. 2. Extraída el 24/VI/2014 desde http:// www.germanlawjournal.com/index.php?pageID $=11 \& \operatorname{artID}=49$ 